\title{
Documenti e immagini del 1977 bolognese
}

Il saggio si propone di presentare alcune considerazioni a margine della pubblicazione del catalogo della mostra documentaria dal titolo Millenovecento77. Quarant'anni dopo: documenti dagli archivi e dalle biblioteche bolognesi.

The aim of this paper is to present some considerations about the catalog of the documentary exhibition entitled Millenovecento 77. Quarant'anni dopo: documenti dagli archivi e dalle biblioteche bolognesi.

Il Movimento del Settantasette ha faticato ad affermarsi come oggetto degli studi storici poiché, sin dalla propria apparizione, si è presentato come una realtà inedita rispetto alle esperienze di azione collettiva sorte a cavallo tra anni Sessanta e Settanta del Novecento [Gagliardi 2017]. In tal senso non appare casuale che nella maggior parte dei lavori storici sull'Italia repubblicana, alle vicende del Movimento siano generalmente destinate poche pagine, riguardanti prevalentemente le più complesse vicende di violenza politica che si sviluppano nei cosiddetti "anni di piombo". Se, all'opposto, ben più vaste sono le produzioni di tipo memorialistico da un lato e giornalistico dall'altro, solo a partire dai primi anni del Duemila vedono la luce lavori che del fenomeno si pongono l'obiettivo di fornire una ricostruzione storica [De Bernardi, Romitelli e Cretella (eds.) 2009; Falciola 2015; Galfrè e Neri Serneri (eds.) 2018]. Confrontandosi con «un caleidoscopio di immagini e una babele di parole» [Falciola 2015, 10], gli studi summenzionati hanno dovuto fare i conti con le molteplici problematicità insite nella natura originale ed eclettica delle fonti che il Movimento del Settantasette ha lasciato in eredità. 


\section{La mostra e la rete}

Quest'ultimo aspetto, la singolarità delle fonti che contraddistingue la cosiddetta "stagione dei movimenti", è il fil rouge del percorso tematico proposto nella mostra dal titolo Millenovecento77. Quarant'anni dopo: documenti dagli archivi e dalle biblioteche bolognesi, tenutasi nella Biblioteca comunale dell'Archiginnasio tra il maggio e il giugno del 2017. L'esposizione si proponeva di presentare al pubblico una variegata selezione di documenti attinta dai vasti giacimenti degli archivi pubblici e privati della città'. Pur mostrando una particolare attenzione per i due momenti chiave del 1977 bolognese - la morte dello studente Francesco Lorusso e il convegno internazionale contro la repressione -, i materiali esposti restituivano la complessità di un dibattito politico, quello della fine degli anni Settanta, contrassegnato dalle riflessioni in materia di lavoro, questione femminile, nuove culture giovanili e rapporto tra studenti, partiti e sindacati. L'organizzazione dell'evento si è altresì configurata come un'occasione di contatto tra i diversi archivi storici della città aderenti all'iniziativa; siffatto confronto, che in fasi successive ha prodotto importanti progetti dedicati alla storia cittadina del Novecento'2, si è infine concretizzato nella costituzione della Rete bolognese archivi del presente. La pubblicazione del catalogo dell'esposizione rappresenta la prima iniziativa ufficiale promossa dalla neocostituita rete che, dalle prime pagine del volume, lancia il proprio manifesto [Gabusi e Maggiorani (eds.) 2019]³.

La mostra è stata promossa dalla Soprintendenza archivistica e bibliografica dell'Emilia-Romagna e dalla Biblioteca comunale dell'Archiginnasio in collaborazione con l'Archivio storico della nuova sinistra "Marco Pezzi", I'Archivio storico Cisl area metropolitana bolognese "Rino Bergamaschi", I'archivio storico "Paolo Pedrelli" della Camera del lavoro metropolitana di Bologna e l'Archivio di Stato di Bologna.

2 In occasione del cinquantennale del 1968, gli archivi storici bolognesi già impegnati nell'iniziativa sul 1977 hanno promosso una mostra documentaria sulle «tracce» del Sessantotto negli archivi della città.

Tra gli aderenti alla rete figurano l'Archivio di Stato di Bologna, I'Archivio storico della Regione Emilia-Romagna - IBC, I'Archivio storico della Città metropolitana di Bologna, il Museo civico del Risorgimento di Bologna, I'Archivio storico "Paolo Pedrelli" della Camera del lavoro di Bologna, I'Archivio storico Cisl area metropolitana bolognese "Rino Bergamaschi", I'Archivio storico della nuova sinistra "Marco Pezzi", la Fondazione Gramsci Emilia-Romagna, l'Archivio storico Unione fotografi organizzati, Archivio Unione donne in Italia (Udi) sede di Bologna, I'Archivio di storia delle donne di Bologna e l'Istituzione Gian Franco Minguzzi. 


\section{2. «Non esisterà uno storico....: il 1977 quarant'anni dopo}

In linea con le ambizioni della mostra, come affermato nella nota introduttiva a firma di Mauro Maggiorani e Marica Tolomelli, il catalogo prosegue nell'opera atta a

dare visibilità al patrimonio documentario, ricco e tipologicamente diversificato, conservato nella nostra città: ciclostilati, relazioni, manifesti, fotografie, video, giornali, fanzine, disegni e dattiloscritti del Movimento, verbali, volantini, materiale fotografico prodotto da istituzioni pubbliche, partiti, sindacati, e tanto altro ancora [Gabusi e Maggiorani (eds.) 2019, 9].

Al testo introduttivo segue un'articolata cronologia del Settantasette bolognese - curata da Davide Fioretto ed Elisabetta Perazzo - che parte dalle occupazioni del 7 e 8 febbraio e si chiude con il corteo che il 25 settembre segna la fine del convegno internazionale contro la repressione. Il percorso tematico proposto nel catalogo, invece, prende le mosse dai primi mesi del 1976, e precisamente dalle proteste contro la riforma promossa dal ministro democristiano Franco Maria Malfatti, quando nella città felsinea si manifestano i primi fermenti. Ed è difatti con la nascita di Radio Alice, risalente al febbraio di quell'anno, che la città assume le sembianze di un laboratorio nel quale si sperimenta una trasformazione delle modalità canoniche che fino alla fine degli anni Settanta avevano caratterizzato la partecipazione alla vita politica del Paese.

Le agitazioni che tra il 1976 e il 1977 investono il capoluogo emiliano hanno un tragico epilogo la mattina dell'11 marzo, quando Francesco Lorusso, studente di Medicina e militante di Lotta continua, viene ucciso in via Mascarella da un proiettile esploso verosimilmente da un uomo al seguito di un'autocolonna delle forze dell'ordine. Nel tracciare le convulse giornate che seguono alla morte dello studente, unitamente agli scatti che immortalano il susseguirsi delle iniziative specularmente promosse dal movimento e dalle organizzazioni partitiche e sindacali, l'oculato incrocio dei documenti selezionati restituisce la complessità di un confronto attraversato da un insieme composito di posizioni.

L'acceso dibattito successivo ai "fatti di marzo", come è noto, oltre a coinvolgere il mondo giovanile e le istituzioni locali, si caratterizza per lo strappo all'interno del mondo intellettuale locale che sino agli anni Settanta era stato vicino - e, in molte sue figure, persino interno - al Partito comunista italiano, e che, nel 1977, sceglie di opporsi alla linea ufficiale del partito. Il volume non manca di occuparsi di questo aspetto, attraverso la pubblicazione di materiali dai quali emerge il vigore di uno scontro che vede protagonisti, in particolare, gli organismi accademici e parte del corpo tecnico e docente. 
Dunque, l'accostamento delle carte provenienti degli archivi della città consente di far affiorare la dialettica tra le complesse stratificazioni sociali e politiche, che caratterizzano la società bolognese degli anni Settanta. È questo il caso del difficile dialogo tra gli studenti e una parte del mondo sindacale: tema oltremodo centrale, seppure ancora poco esplorato, considerato che la questione delle occupazioni

fu uno dei principali terreni di confronto tra sindacato e movimento studentesco. Fallito il dialogo con il Movimento, il sindacato trovò nelle leghe dei disoccupati costituite attingendo alle liste speciali di collocamento previste dalla legge 285/1977 (Provvedimenti per l'occupazione giovanile) lo strumento per unire le masse giovanili e canalizzarle nell'orbita della Federazione unitaria [Gabusi e Maggiorani (eds.) 2019, 61].

Tra i volantini, dai quali emergono i riverberi della polemica tra gli studenti e le istituzioni locali, e le foto delle piazze bolognesi, ricolme di giovani provenienti da tutta Italia, il percorso espositivo si conclude con il materiale sul convegno contro la repressione celebrato a Bologna tra il 23 e il 25 settembre 1977.

Arricchiscono la pubblicazione Fabrizio Billi e Valentina Gabusi che introducono il materiale d'archivio attinente ai canali comunicativi adottati dal Movimento. Il primo dei due contributi riguarda le cosiddette "fanzine", ovvero

fogli dalla periodicità irregolare, di cui solitamente uscivano pochi numeri o anche uno solo, realizzati in offset, tecnica di stampa diffusasi in Italia all'inizio degli anni Settanta, che permetteva una composizione libera, in cui si potevano combinare a piacimento, grazie a forbici e colla, foto, testi dattiloscritti e scritte a mano, disegni, fotomontaggi [Gabusi e Maggiorani (eds.) 2019, 85].

Oltre alle più note "A/Traverso" e "Zut", nel volume vengono raccolte le prime pagine di numerose riviste i cui titoli sono la sintesi più rappresentativa di quel "maodadaismo" che, nel giudizio di Billi e Gabusi, «consiste nella politica più la scrittura e la comunicazione, il tutto volto ad affermare la propria soggettività e ad esprimere i propri bisogni» [Gabusi e Maggiorani (eds.) 2019, 86] .

In un altro contributo, Billi e Gabusi analizzano i manifesti murali, rilevando come la scarsa originalità di queste forme espressive sia connessa alla mancanza di quella carica innovativa che caratterizza, invece, fanzine e volantini. Fatta eccezione per una parte di materiali realizzati dagli animatori di Radio Alice, infatti,

"Vogliamo tutto", "Complotto", "Désir", "Red/Azione diretta", "Che cento fiori sboccino che cento lotte esplodano per disarmare il potere", "La luna o il dito", "L'unanimità", "Il limone a canne mozze", "Il complotto", "Oask", "Groucho", "Caccolone Brivido", "La scimmia", "11 marzo", "Giornale dei non garantiti (praticamente tutti)", "Il Resto del Crimine", "Zangherì, zangherà dicci la verità". 
gli stili grafici del Movimento sembrano in questo caso rientrare nei canoni tradizionali, al punto da apparire non dissimili da quelli impiegati dai partiti dell'arco costituzionale.

In chiusura, due saggi di Luca Falciola e Luca Chiurchiù contestualizzano sul piano storiografico la riflessione sull'esperienza del Movimento nel capoluogo emiliano. Luca Falciola, in particolare, ribadendo come il Movimento si caratterizzi per una ineliminabile natura evanescente, riprende l'auspicio di sottrarsi al giudizio degli storici lanciato dagli studenti proprio in quello stesso 1977:

non esisterà uno storico, non tollereremo che esista uno storico, che assolvendo una funzione maggiore del linguaggio, offrendo i suoi servizi alla lingua del potere, ricostruisca i fatti, innestandosi sul nostro silenzio, silenzio ininterrotto, interminabile, rabbiosamente estraneo [autori molti compagni 1977, 9].

Sotto questo aspetto, si può concordare con Falciola sul fatto che far diventare un «reperto museale» il Movimento che «si consumò nel qui ed ora assoluto», non può che apparire come un ironico «scherzo del destino». Tuttavia, proprio seguendo le suggestioni da lui offerte nel saggio, si deve rilevare come aver intrappolato il Movimento nelle teche di una mostra, con la riscoperta e l'analisi di nuove fonti documentarie, è un'operazione che ha permesso di delineare quella realtà come un importante fatto storico che «emerge in forma tridimensionale come un ciclo di contestazione dai caratteri originali» (ancorché connesso alla lunga stagione dei movimenti iniziata alla fine degli anni Sessanta) [Gabusi e Maggiorani (eds.) 2019, 137].

Nel secondo saggio, a firma di Luca Chiurchiù, l'attenzione si concentra sull'«arcipelago» della vasta produzione di riviste e giornali espressione del Movimento. La metafora adoperata appare profondamente calzante giacché si connette alla più complessiva necessità di superare l'immagine stereotipata, e per questo fuorviante, che descrive il Movimento del Settantasette come una realtà dall'anima "bifida", ossia

un enigmatico fenomeno dove da una parte stava la violenza, di piazza o meno, diffusa o organizzata, e dall'altra, invece, un fermento culturale e creativo a dir poco notevole, quello della cosiddetta ala desiderante, votata alla trasformazione della propria esistenza attraverso l'arte, il gioco e, spesso, le droghe [Gabusi e Maggiorani (eds.) 2019, 144].

Seguendo invece le ricostruzioni offerte dalla storiografia più recente, Chiurchiù parla di «due oceani che comunicarono più volte tra loro, e che le correnti che li attraversarono furono in molti casi le medesime, senza soluzione di continuità». Eppure, e questo appare probabilmente come il limite più evidente della pubblicazione, della «compromissione» tra le due anime vi è poca traccia quantunque, 
come scrive Gagliardi, «la violenza politica - è indubbio - attraversò il '77 e lo segnò indelebilmente» [Gagliardi 2017, 34]. Nel saggio viene offerta un'attenta analisi della produzione di riviste realizzate dal Movimento: una fonte preziosa, quest'ultima, che proprio grazie alla sua natura originale, di documento realizzato per parlare al presente senza nessuna velleità di arrivare ai posteri, rivela una volta di più la natura magmatica della protesta studentesca. Difatti, dalla panoramica proposta da Chiurchiù, emerge la convivenza tra esperimenti frutto di una creatività pensata per essere compresa solo dagli studenti ed esperienze che, al contrario, intorno alla scrittura come pratica rivoluzionaria tentarono di avviare una vera e propria riflessione politica.

In conclusione, come confermano i più recenti studi sull'argomento, il 1977 bolognese ha costituito uno spartiacque che ha profondamente segnato la storia della città. In tal senso, la pubblicazione esaminata, valorizzando ambiti documentari di varia natura, offre indubbiamente un contributo utile a superare quel «destino storiografico infelice», come scriveva Sandro Bellassai, che per molti anni ha schiacciato la storia del Movimento fra il "lungo Sessantotto" e il caso Moro, facendolo divenire, nelle diverse interpretazioni, epilogo del primo o prologo del secondo. [Bellassai 2010, 216]. L'auspicio, già lanciato da Gagliardi, è che lo studio del Settantasette possa continuare ad avanzare «con rigore e acribia, non meno che con passione e coinvolgimento» [Gagliardi 2017, 13], con l'obiettivo di superare quel divario che, come affermato da Giovanni De Luna, caratterizza gli anni Settanta per «troppa memoria e poca storia. Troppi ricordi e pochi documenti, troppi sentimenti e poca filologia» [De Luna 2009, 162].

\section{Bibliografia}

autori molti compagni 1977. bologna marzo 1977 ... fatti nostri ..., Verona: Bertani editore

Bellassai S. 2009. Un trauma che si chiama desiderio. Per una storia del Settantasette a Bologna, in De Bernardi, Romitelli, Cretella (eds.) 2009

De Bernardi A., Romitelli V. e Cretella C. (eds.) 2009. Gli anni Settanta Tra crisi mondiale e movimenti collettivi, Bologna: Archetipo libri

De Luna G. 2009. Le ragioni di un decennio. 1969-1979. Militanza, violenza, sconfitta, memoria, Milano: Feltrinelli

Falciola L. 2015. Il Movimento del 1977 in Italia, Roma: Carocci

Gabusi V. e Maggiorani M. 2019. Millenovecento77. Quarant'anni dopo: documenti dagli archivi e dalle biblioteche bolognesi, Bologna: Biblioteca comunale dell'Archiginnasio 
Gagliardi A. 2017. Il '77 tra storia e memoria, Roma: Manifestolibri

Galfrè M. e Neri Serneri S. (eds.) 2018. Il movimento del '77. Radici, snodi, luoghi, Roma: Viella

\section{Risorse}

Manifesto della Rete bolognese archivi del presente http://www.archiviodistatobologna.it/it/novita-avvisi/novita/rete-bolognese-archivipresente 
